Originalien

Psychotherapeut 2020 - 65:197-204 https://doi.org/10.1007/s00278-020-00411-3 Online publiziert: 25. März 2020

(c) Der/die Autor(en) 2020

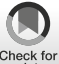

Loni Brants ${ }^{1}$ Katrin Schuy ${ }^{1}$ Simone Dors ${ }^{1}$ Marie Horzetzky ${ }^{1}$ Heinrich Rau ${ }^{2}$. Peter L. Zimmermann ${ }^{2}$. Andreas Ströhle ${ }^{1}$. Stefan Siegel ${ }^{1}$

'Klinik für Psychiatrie und Psychotherapie, Charite - Universitätsmedizin Berlin, Campus Charité Mitte, Berlin, Deutschland

${ }^{2}$ Psychotraumazentrum, Bundeswehrkrankenhaus Berlin, Berlin, Deutschland

\title{
Integrativer Modellentwurf zu Coping und Abwehr ehemaliger BundeswehrsoldatInnen
}

ausgeschieden sind, umso mehr (Siegel et al.2017). Neben teils traumatischen Erlebnissen im Ausland und Diskriminierungserfahrungen in Deutschland sehen sich (ehemalige) Bundeswehrsoldaten im Allgemeinen mit einer Vielzahl von Herausforderungen konfrontiert, die ein extrem hohes Maß an Anpassungsfähigkeit erfordern. Hierzu zählen die Eingliederung in das Militärsystem, Kriegserfahrungen im Ausland, dieWiedereingliederung in den militärischen Berufsalltag und das Familienleben nach der Rückkehr aus dem Auslandseinsatz, das endgültige Verlassen des Militärs, die Wiedereingliederung in das zivile Leben und ggf. der Umgang mit Symptomen einer Traumafolgestörung (Schuy et al. 2019; Brants et al. 2018). Wissenschaftliche Untersuchungen mit dem Fokus auf Möglichkeiten der bewussten und unbewussten Bewältigung in dieser Gruppe existierten gemäß dem Wissen der Autoren des vorliegenden Beitrag bislang nicht.

\section{Coping und Abwehr als Bewältigungsformen} geringen Therapieinanspruchnahme verbunden (Wittchen et al. 2012). Im Gegensatz zum breiten Kenntnisstand über die psychische Gesundheit beispielsweise US-amerikanischer SoldatInnen ist über den Umgang mit belastenden Ereignissen und psychischer Krankheit aktiver deutscher BundeswehrsoldatInnen kaum etwas bekannt (Kowalski et al. 2012). Dies gilt für die Bewältigungsmechanismen ehemaliger BundeswehrsoldatInnen, die inzwischen aus dem militärischen System logischer Forschung (Übersicht: Schwarzer 1998). Die in der Folge entwickelten Kategoriensysteme und Messinstrumente, wie der bis heute genutzte Coping Orientation to Problems Experienced Scale (COPE; Kato 2015; Carver et al. 1989), machten es möglich, Coping-Mechanismen durch Selbstauskunft zu erfahren und Coping-Konzepte zu entwickeln. Die Rolle individueller Persönlichkeitseigenschaften und (biografischer) Motive wurde dabei jedoch oft vernachlässigt (Steffens und Kächele 1988). Lazarus (2000) selbst räumte ein, dass unbewusste Intentionen auf diese Weise kaum abgebildet würden. Obwohl die Forschungsergebnisse der letzten Jahre den hohen Anteil unbewusster, intuitiv verlaufender Prozesse insbesondere bei komplexen, schnell zu treffenden Entscheidungen untermauern (Horr et al. 2014; Gigerenzer und Kober 2009), hat sich daran bisher wenig geändert.

Abwehrmechanismen können „als unbewusste, vorwiegend kognitiv-erfahrungsbezogene Prozesse, die eine Einengung oder Verzerrung von intersubjektiver Realität, Selbstwahrnehmung oder beidem implizieren“, definiert werden (Beutel 1990, S. 1-12). Psychoanalytiker verschiedener Strömungen interpretieren Abwehr auf unterschiedliche Weise, wobei jede Definition das Risiko einer zu großen Vereinfachung mit sich bringt. In ihrem 600-seitigen Buch über den aktuellen Stand von Theorie und Forschung zu Abwehrmechanismen, betonen Hentschel et al. (2004) zu Recht die Komplexität des Themas. Nach der 
Theorieentwicklung von Sigmund Freud und deren Weiterentwicklung durch seine Tochter Anna Freud (2006 [1936]) wurde das Konzept der Abwehr durch die verschiedenen Strömungen innerhalb der psychoanalytischen Lehre um intrapsychische und interpersonale Perspektiven ergänzt. Hierbei wurde die Beschaffenheit der individuellen psychischen Struktur und ihrer entsprechenden Mechanismen u.a. im Rahmen der psychodynamischen Diagnostik hervorgehoben (Cierpka 2014). Im Laufe der letzten Jahrzehnte hat es durch Autoren wie Vaillant (1971, 1992), Laughlin (1979) und König (1997) immer wieder Taxonomiebestrebungen gegeben, wobei sich Zahl und Einteilung der Mechanismen je nach Autor deutlich unterschieden (Seiffge-Krenke 2017). Relative Einigung scheint in der psychoanalytischen Lehre darüber zu bestehen, dass Abwehr- und auch Coping-Mechanismen klare Ich-Funktionen haben bzw. sind, wobei die Abwehr zur intrapsychischen Regulation, das Coping eher zu realer Anpassung und Problemlösung eingesetzt werden (Steffens und Kächele 1988). In anderen Worten: Erst die entsprechende (unbewusste) Abwehr ermöglicht ein erfolgreiches (bewusstes) Coping (Cierpka 2014).

Zahlreiche namenhafte Autoren haben sich in den letzten Jahrzehnten wiederholt kritisch $\mathrm{zu}$ einer expliziten Trennung von Coping/Bewältigung und Abwehr geäußert (Cierpka 2014; Steffens und Kächele 1988; Beutel 1990). So schreiben Steffens und Kächele bereits 1988: „Wir halten es ... für sinnvoll, eine strikte Trennung von Bewältigung und Abwehr aufzugeben. Beide Vorgänge ergänzen sich, schließen sich keineswegs alternativ aus.“ Trotzdem kam es in der Vergangenheit wiederholt $\mathrm{zu}$ „Verleugnungsbemühungen der Verwandtschaftsbeziehungen" von Coping- und Abwehrkonzepten (Cierpka 2014; Steffens und Kächele 1988; Beutel 1990). Auch jetzt deuten der Einsatz von Coping-Selbst-Rating-Inventaren in der klinisch-psychiatrischen Praxis und Wissenschaft auf der einen bzw. die Fokussierung auf unbewusste Konflikte und Abwehrmechanismen psychoanalytischer Therapeuten und Wissenschaftler auf der anderen Seite auf ein bis zum heutigen Tag noch sehr einseitiges Vorgehehen hin. Ein praxisnahes, schulenübergreifendes und integratives Modell zur Identifikation und Darstellung von Coping- und Abwehrmechanismen bei Traumafolgestörungen existiert gemäß Wissen der Autoren bislang nicht. Dies kann verwundern, da Mechanismen zur Bewältigung aversiver Situationen (z.B. Sport oder Rauschmittelkonsum) und/ oder unbewusste Abwehrmechanismen (z. B. Rationalisieren oder Spalten) in der Diagnostik, Therapieplanung und Prognoseeinschätzung von Psychotherapeuten verschiedener Schulen eine wichtige Rolle spielen. Dies gilt in besonderem Maß für Patienten mit Traumafolgestörungen, in deren Behandlung Ichstützende, affektregulierende Maßnahmen von großer praktischer Bedeutung sind.

\section{Ziel der Arbeit}

Ziel der dargestellten Untersuchung war es, durch persönliche, offene Interviews einen Einblick in die Abwehr- und Coping-Mechanismen ehemaliger BundeswehrsoldatInnen mit Einsatzerfahrung unter besonderer Berücksichtigung ihrer militärischen Laufbahn zu erhalten. Somit war es möglich, anhand einer Gruppe von Menschen, die aufgrund ihrer beruflichen Laufbahn sowie schwer zu verarbeitender Erfahrungen ein hohes $\mathrm{Maß}$ an Anpassungsleistungen erbringen mussten, den ersten Entwurf für ein schulenübergreifendes Instrument zur Identifikation und Darstellung bewusster und unbewusster Bewältigungsmechanismen zu entwickeln.

\section{Material und Methode}

\section{Rekrutierung und "sampling"}

Die Rekrutierung erfolgte über eine projekteigene Webseite und das Bundeswehrkrankenhaus Berlin. Mit 98 potenziellen Teilnehmern wurden (fern-) mündliche Kurzinterviews zur Erfragung der soziodemografischen Daten geführt, 43 wurden in der Folge nacheinander $\mathrm{zu}$ offenen Interviews eingeladen. Es wurden ehemalige Einsatzsoldaten der
Bundeswehr mit und ohne Symptomatik sowie mit und ohne Inanspruchnahme psychosozialer Leistungen ausgewählt, um im Sinne des ,theoretical sampling“ eine kontrastierende Auswahl (Corbin und Strauss 2008) und möglichst hohe Variabilität zu erreichen (•Tab. 1). Jedes Interview wurde zeitnah im Rahmen einer teaminternen Forschungsintervision hinsichtlich Ablauf, Inhalt und Gegenübertragung nachbesprochen, mit vorangehenden Interviews verglichen und das weitere Sampling festgelegt. Nach insgesamt 43 Interviews war eine theoretische Sättigung erreicht. Bezugnehmend auf die dargestellte Fragestellung wurden 16 Interviews der detaillierten Textanalyse unterzogen. Diese waren anhand kontrastierender Merkmale (Herkunft, Alter, Geschlecht, Beziehungsstatus, Kinderanzahl, Ausbildungsstand, militärischer Rang, Organisationsbereich, Einsatzland und -dauer, psychische Symptombelastung, Psychotherapieerfahrung, Wehrdienstbeschädigungsantrag) ausgewählt worden. Die daraus erarbeiteten Konzepte zur Qualitätssicherung wurden mit den verbleibenden 27 Interviews abgeglichen.

\section{Datenerhebung und -analyse}

Die Datenerhebung erfolgte in offenen, persönlichen Interviews. Die Interviews wurden mit einer Frage nach dem beruflich-militärischen und privaten Werdegang begonnen; der weitere Gesprächsverlauf ergab sich aus den Schilderungen des Befragten bzw. Nachfragen des Interviewers. Bewusste Coping-Mechanismen (z. B. Alkoholkonsum, Sport etc.) wurden im Gesprächsverlauf teils unaufgefordert berichtet, teils zu einem späteren Zeitpunkt direkt erfragt. Unbewusste Coping- und Abwehrmechanismen wurden aus den sehr detailliert beschriebenen Situations- und Handlungsabläufen der Teilnehmer abgeleitet (z. B. Rationalisierung, Verleugnung etc.) oder während des Interviews festgestellt (z. B. Affektisolation, Dissoziation, Entwertung etc.).

Die Interviews wurden im MP3-Format aufgezeichnet und verschriftlicht. Für diese Verschriftlichung wurde ein einfaches Transkriptionssystem gewählt (buchstäbliche Übertragung unter Weg- 
lassung von dialektalen Eigenheiten, Funktionswörtern und Verständnissignalen sowie unter Sprach- und Interpunktionsglättung (Dresing und Pehl 2015)).

Der methodische Auswertungsprozess in Anlehnung an das Prinzip der Grounded Theory und der thematischen Analyse beinhaltet die iterative Generierung von Hypothesen und Entwicklung neuer Modelle. Entsprechend Chapman et al. (2015) wurde sich dem Material iterativ über induktive Erarbeitung, Deduktion und Validierung angenähert, um eine im Material verankerte und daraus erwachsene Theorie zu entwickeln. Die Datenerhebungs- und Analyseprozesse wurden weitergeführt, bis eine „Sättigung" eintrat, d.h., sich keine neuen Erkenntnisse aus der Datenerhebung und Analyse mehr ergaben. Diese Herangehensweise entsprach den von Guest et al. (2012) genannten Schritten:

1. Kennenlernen des Materials,

2. Identifizierung von Themenbereichen,

3. Identifizierung von Strukturen und

4. Aufbau eines theoretischen Modells, basierend auf den gewonnenen Erkenntnissen.

Der erste Schritt bestand aus dem Kennenlernen des Materials durch das wiederholte Hören der Interviews, Lesen der Transkripte sowie Erstellen von Notizen und Querverweisen. Erste Codes wurden vergeben, um die Bedeutung von Abschnitten und Beobachtungen festzuhalten oder zusammenfassen. Im zweiten Schritt erfolgte die Identifizierung von Themenbereichen, indem einander ähnelnde oder miteinander verbundene Codes kombiniert, kontrastiert und zusammengefügt werden. Der dritte Schritt enthielt die Überprüfung und Analyse der gefundenen Bereiche zur Identifizierung von dahinter liegenden thematischen Strukturen und Reduktion übergeordneter Themenbereiche anhand neuer Rohdaten aus dem laufenden Prozess der Datenerhebung und -analyse. Der vierte und letzte Schritt bestand im Aufbau eines theoretischen Modells, basierend auf den im Material gefundenen Mechanismen unter ständiger Gegenprüfung mit neuem Material sowie nun auch unter

Psychotherapeut 2020 65:197-204 https://doi.org/10.1007/s00278-020-00411-3

(c) Der/die Autor(en) 2020

L. Brants · K. Schuy · S. Dors · M. Horzetzky · H. Rau · P. L. Zimmermann · A. Ströhle · S. Siegel

Integrativer Modellentwurf zu Coping und Abwehr ehemaliger BundeswehrsoldatInnen

\section{Zusammenfassung}

Verglichen mit dem profunden Wissen über die Erfahrungen und Bedürfnisse US-amerikanischer Soldaten, ist der Kenntnisstand über die psychische Gesundheit und die Bewältigungsmechanismen aktiver deutscher BundeswehrsoldatInnen eher gering. Über ehemalige EinsatzsoldatInnen, die inzwischen aus dem militärischen System ausgeschieden und Teil der deutschen Zivilgesellschaft geworden sind, ist noch viel weniger bekannt. Ziel der Pilotstudie war die Identifikation und Darstellung von Coping- und Abwehrmechanismen ehemaliger BundeswehrsoldatInnen mithilfe einer methodisch-strukturierten, qualitativen Datenerhebung. Zu diesem Zweck wurden 43 Interviews mit ehemaligen EinsatzsoldatInnen geführt, transkribiert und der thematischen Analyse unterzogen. Die identifizierten Coping- und Abwehrmechanismen wurden den Bereichen Verhalten, Beziehung, Emotion, Reflexion und Zeit zugewiesen und modellhaft in Achsenform veranschaulicht. Durch die bewusste Synthese von kognitiv-behavioralen und psychoanalytischen Perspektiven konnte ein differenziertes Bild erstellt sowie ein eigenständiges, schulenübergreifendes, integratives Achsenmodell zur Darstellung der bewussten und unbewussten Bewältigung belastender Erfahrungen entwickelt werden.

Schlüsselwörter

Qualitative Forschung · Psychische Gesundheit - Verhalten - Emotionen . Veteranen

\section{Draft of an integrative model on coping and defence of former soldiers in the German Armed Forces}

\section{Abstract}

Compared to the profound knowledge about the experiences and needs of American soldiers, in Germany the level of knowledge on mental health and coping mechanisms of active soldiers in the German Armed Forces (GAF) is relatively limited. Even less is known about former soldiers who are no longer on active duty and who, in the majority of cases, have become part of the German public healthcare system after discharge from the military. The aim of the pilot study was the identification and presentation of the coping and defence mechanisms of former GAF soldiers by using methodically structured, qualitative data collection. For this purpose, 43 interviews with former soldiers were conducted, transcribed and thematically analyzed. The coping and defence mechanisms identified were assigned to the areas of behavior, relationship, emotion, contemplation and time and visualized as an axis model. By consciously synthesizing cognitive-behavioral and psychoanalytical perspectives, it was possible to create a differentiated picture and develop an independent, integrative model across psychotherapeutic school boundaries to illustrate the conscious and unconscious coping with traumatic experiences.

\section{Keywords}

Qualitative research - Mental health . Behavior - Veterans · Interpersonal relations
Einbezug aktueller Literatur und bestehender Modelle.

\section{Ergebnisse}

Insgesamt zeigte sich eine sehr große Zahl und Bandbreite an beschriebenen, benannten und beobachteten Abwehrund Coping-Mechanismen innerhalb der befragten Gruppe und auch innerhalb der einzelnen Interviews. So konnten im ersten Schritt der thematischen Analyse (nach Guest et al. 2012; Chapman et al. 2015) 1960 Textabschnitte extrahiert werden, die Formen der bewussten und unbewussten Konfliktbewältigung enthielten und sich wiederum zu 89 Codes zusammenfassen ließen. Diese 89 Codes konnten im zweiten Analyseschritt entsprechend ihrem Inhalt, Motiv und ihrer Funktion 15 übergeordneten Copingund Abwehr-Bereichen zugeteilt wer- 
Tab. 1 Merkmale der Stichprobe $(n=43)$ zur VeteranInnenbefragung

Einbezogene Interviews 43 (Anzahl, $n$ )

Davon weiblich

4

Davon männlich

Alter (Jahre)

Altersspanne 29-69

Altersdurchschnitt 40,4

Standardabweichung

$\pm 12,3$

Erwerbsstatus (Anzahl, $n$ )

Erwerbstätig

25

Arbeitssuchend

4

Sonstiges (z. B. krank, Student) 14

Dienstgrad (Anzahl, $n$ )

Mannschaft

6

Unteroffizier

30

Leutnant

3

Hauptmann

3

Stabsoffizier

1

Organisationseinheit (Anzahl, $n$ )

Heer

23

Marine

Luftwaffe

6

Streitkräftebasis (SKB)

2

Zentraler Sanitätsdienst

5

(ZSanDst)

Wehrverwaltung

2

Teilnahme an Auslandseinsätzen (Einsatztage)

90-300

90-2190

301-600 27

Mehr als 600

8

Gesundheitsstatus (Anzahl, $n$ )

Diagnose einer psychischen Erkrankung ${ }^{\mathrm{a}}$

Wehrdienstbeschädigung $^{\mathrm{a}} \quad 19$

Symptome einer psychischen $\quad 9$

Erkrankung ohne Diagnose ${ }^{a}$

Ohne Symptome einer psychi- $\quad 10$ schen Erkrankung ${ }^{\mathrm{a}}$

${ }^{a}$ Nach Angaben der Teilnehmerlnnen

den. Im Rahmen des dritten Schritts, der Überprüfung und Analyse der gefundenen Bereiche zur Identifizierung von dahinter liegenden thematischen Strukturen, konnten die bestehenden $15 \mathrm{Be}$ reiche auf die fünf übergeordneten Themenbereiche „Verhalten“, „Beziehung“, „Emotion“, „Reflexion“ und „Zeit" reduziert werden. Im vierten Schritt wurden bereits bekannte theoretische Grundan- nahmen mit den neu gefundenen Daten und Strukturen abgeglichen und in ein Modell integriert. Dabei wurden die 5 gefundenen Bereiche entsprechend ihrer Ausprägung von Minus (-) nach Plus (+) grafisch in einem Achsenmodell dargestellt. Die Achsen illustrieren die wertneutralen, sich gegenüberliegenden Pole eines Kontinuums.

Der Großteil der Befragten zeigte im Rahmen belastender Situationen Coping- und Abwehrmechanismen mehrerer Achsen und innerhalb dieser mitunter auch zu beiden Polen tendierende Ausprägungen sowie dominierende, sich wiederholende Kernmechanismen eines bestimmten Achsenpols. In der grafischen Darstellung finden sich an beiden Seiten des Kontinuums erklärende Poldefinitionen und jeweils darunter ausgewählte Beispielzitate aus dem Interviewmaterial (• Abb. 1).

Insgesamt wurde bei allen Befragten eine Häufung bewusster Coping-Mechanismen im hohen Aktivitätsbereich sowie eine große, teils mehr teils weniger bewusste Notwendigkeit, in der sozialen Gruppe zu bestehen und sich mit dieser verbunden zu fühlen, deutlich. Der große Wunsch nach Nähe und Verbundenheit führte mitunter zum Zurückstellen und zum Verdrängen eigener Bedürfnisse oder seltener in stark abgewehrter Form zum totalen Rückzug, dem Entwerten oder Vermeiden von intimen Situationen und den damit verbundenen Gefühlen. Emotionale Reflexion im Sinne von Wahrnehmung, Einordnung und Mitteilung von Gefühlen zeigte sich insgesamt weniger deutlich, bzw. kam es wiederholt zu Situationen, in denen emotionales Erleben (auch innerhalb der Interviews) verdrängt, rationalisiert, bagatellisiert oder geleugnet wurde. Dies traf jedoch nicht auf die Gruppe der Befragten mit Psychotherapieerfahrung zu, die ihre emotionalen Erfahrungen meist entsprechend offen verbalisierte.

\section{Diskussion}

\section{Einordnung der Ergebnisse}

Bei der Darstellung der Ergebnisse in Achsenform handelt es sich um das erste, aus dem Material gewonnene Modell zur Veranschaulichung eines integrativen, Psychotherapieschulen und -strömungen verbindenden Denkansatzes. Im Rahmen diagnostischer Gespräche soll so ohne den Einsatz weiterer Fragebogen und unabhängig von der psychotherapeutischen Prägung eine erste Einschätzung der bewussten und unbewussten Bewältigungsmöglichkeiten des Gegenübers getroffen werden können. In Teilbereichen lehnt sich das gewählte Achsenmodell an bereits bestehende Coping-, Abwehr- und Strukturmodelle an, wie beispielsweise die Strukturbestimmung innerhalb der operationalisierten psychodynamischen Diagnostik (OPD), oder aktuelle Coping-Inventare, wie den COPE, auf den im nächsten Absatz eingegangen wird. Eine nachträgliche Anpassung an diese Konstrukte erschien nicht zielführend, insbesondere weil sich die meisten Coping-Inventare ausschließlich mit bewussten Vorgängen beschäftigen, die psychodynamische Diagnostik hingegen nur selten eine explizite Erhebung bewusster CopingMechanismen/-Stile vorsieht. Daher wurde sich für den Versuch der Integration verschiedener psychologischer Perspektiven entschieden. Entsprechend den Prinzipien qualitativer Forschung rückten das subjektive Erleben des Individuums und die von ihm genannten und gezeigten Mechanismen unter Einbezug seiner individuellen Motive in den Vordergrund.

Überschneidungen des Achsenmodells wurden, wie bereits erwähnt, mit den Skalen des von Carveret al. (1989) entwickelten COPE gefunden. Das Messinstrument ist ein theoriebasiertes SelfRating-Inventar und umfasst die Kategorien „positive reinterpretation and growth“, „mental disengagement“, „focus on and venting of emotions", „use of instrumental social support", „active coping“, „denial“, „religious coping“, "humor“, „behavioral disengagement", "restraint", „use of emotional social support“, „substance use“, „acceptance“, "suppression of competing activities" und „planning“. Bis auf den Bereich "turning to religion" fanden sich alle Kategorien, teils wörtlich, teils in abgewandelter Form, auch innerhalb der hier entwickelten Codes und Bereiche 


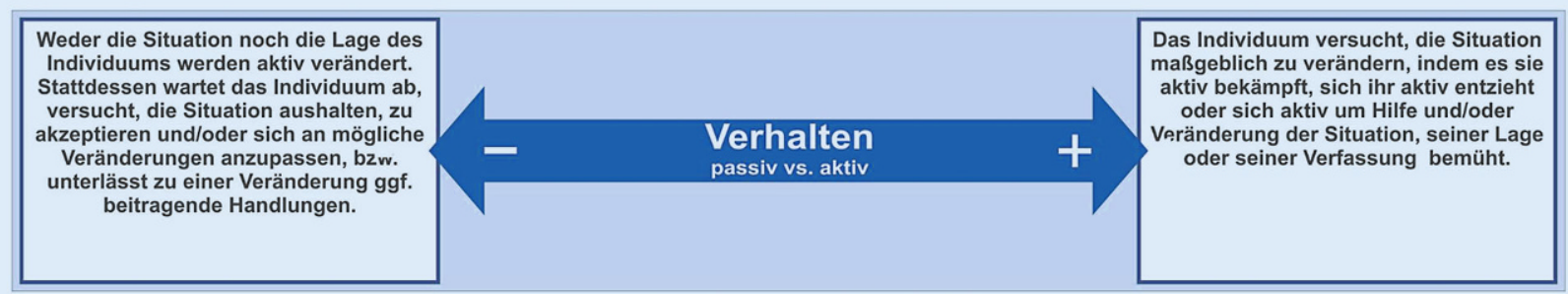

Zitat: „Ab Oktober war ich dann wieder zu Hause geblieben. Da war es eine Woche. Dachte ich mir, ja gut, vielleicht dauert es halt diesmal ein bisschen länger, bis man sich fängt. Aus zehn Tagen sind dann fünf Wochen geworden. "
Zitat: „Und so blieb uns nichts anderes über, als dass ich gesagt habe ,Jungs/', also, wir hatten alle so viel Adrenalin, ne, schießt einfach mal auf die Wand. "und Schießen schafft Sicherheit. Ne, ein bisschen Adrenalin abbauen. ,Vielleicht hauen die ab, weil wir eine Feuerüberlegenheit haben. ${ }^{~}$

\begin{tabular}{|c|c|c|}
\hline $\begin{array}{c}\text { Das Individuum vermeidet enge } \\
\text { Beziehungen, reagiert auf intime } \\
\text { Situationen mit Rückzug oder wehrt } \\
\text { Nähewünsche mittels Entwertung und } \\
\text { Leugnung ab. Die eigenen Interessen } \\
\text { stehen im Vordergrund, Probleme } \\
\text { müssen allein gelöst, die eigene } \\
\text { Autonomie unbedingt erhalten werden. }\end{array}$ & $\begin{array}{c}\text { Das Individuum kann anhaltend Nähe } \\
\text { suchen, finden, aushalten und von ihr } \\
\text { profitieren. Es vertraut sich anderen } \\
\text { an, kann um Hilfe bitten, diese } \\
\text { annehmen und auch spenden. Alte } \\
\text { Beziehungen werden um jeden Preis } \\
\text { erhalten, neue soziale Netzwerke } \\
\text { aufgebaut. Eigene Interessen werden } \\
\text { denen des Umfelds untergeordnet. }\end{array}$ \\
\hline
\end{tabular}

Zitat: „Und leider, wie es erwartet war, ging es dann eigentlich schon los relativ mit den ersten/also, ich habe schon sondiert, wie es wäre mit einer Scheidung. Warum, ich weiß nicht, ich hatte keinen Bock aufNähe. Das war mir alles zu viel."
Zitat: „Heute habe ich immer noch Kontakt mit Kollegen und Kameraden aus der Zeit von 99, worauf ich auch sehr stolz bin, keine Frage. Weil das sind wirklich Leute, die kann ich jetzt anrufen, die kommen her und ich will nicht sagen, "die machen dann was", aber die sind dann für einen da."

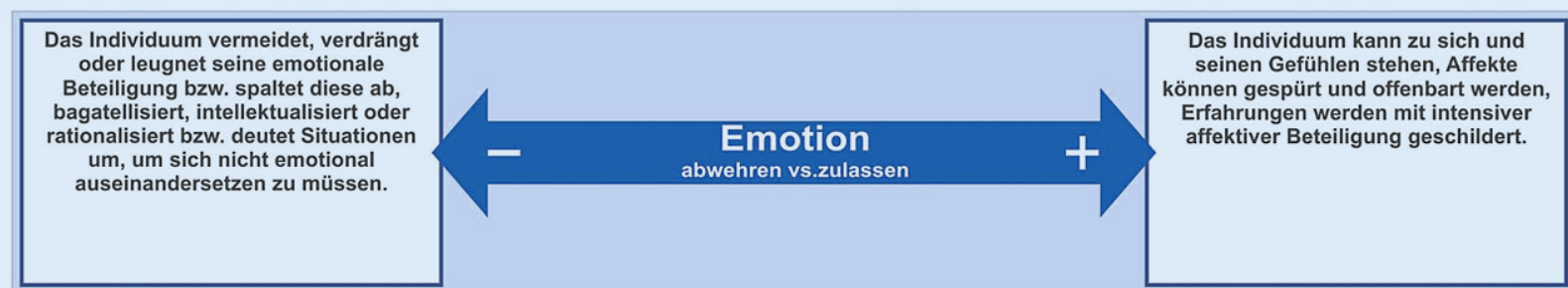

Zitat: ,Ich habe mich meistens immer betäubt mit viel Sport. Ne? Ausgepowert sein, damit ich auch ja ins Bett komme und auch direkt schlafe, ne. (...) Unbewusst sucht man sich sehr hohe (...) Möglichkeiten oder sehr viele Möglichkeiten, sich zu betäuben. (....“

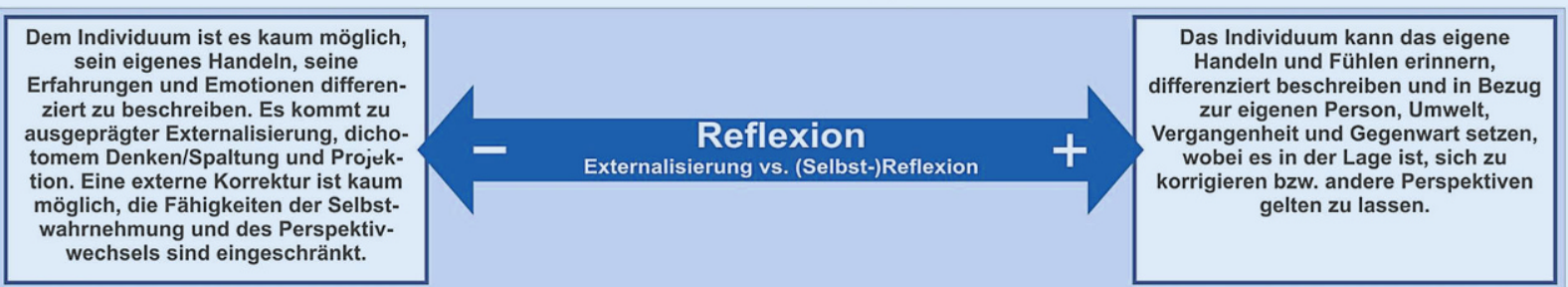

Zitat: „Diese Gleichgültigkeit den Fahrgästen gegenüber, ja, Verspätung und hier und da und dann drängeln die Fahrgäste und all so ein Scheiß, (...) rotzunfreundliche Busfahrer, weil die auch schon genervt waren, von ihrem Job, keine unfreundliche Busfahrer, weil die auch schon genervt waren, von ihrem Job, keine
Ahnung. Jedenfalls, das ging mir so auf den Senkel, wo ich mir dann schon gedacht habe, "Mensch, Alter, dieses beknackte Volk da draußen (...)"
Zitat: „Und für mich war das so eine Kack-Situation, weil irgendwie hat keiner berücksichtigt, dass ich da liege und schlafe. Die anderen sind wohl vorher schon wach geworden und haben sich einfach dem Feuerkampf angeschlossen, aber ich habe mich ziemlich alleine gefühlt. Ich habe keinen gesehen, ich habe zwar irgendwas schießen hören, lag aber im Endeffekt ohne Ausrüstung da und hatte halt Schiss (...)

Zitat: „Aber ich bin dafür bekannt, dass ich auch Asche auf mein Haupt streue und sage: Leute, das war Käse'. Und ich finde auch einen Wert gut, den habe ich auch im Kosovo gehabt, wenn ich in der Früh in den Spiegel geguckt habe und sage: "das, was du da gestern gemacht hast, das war Scheiße". Ja."

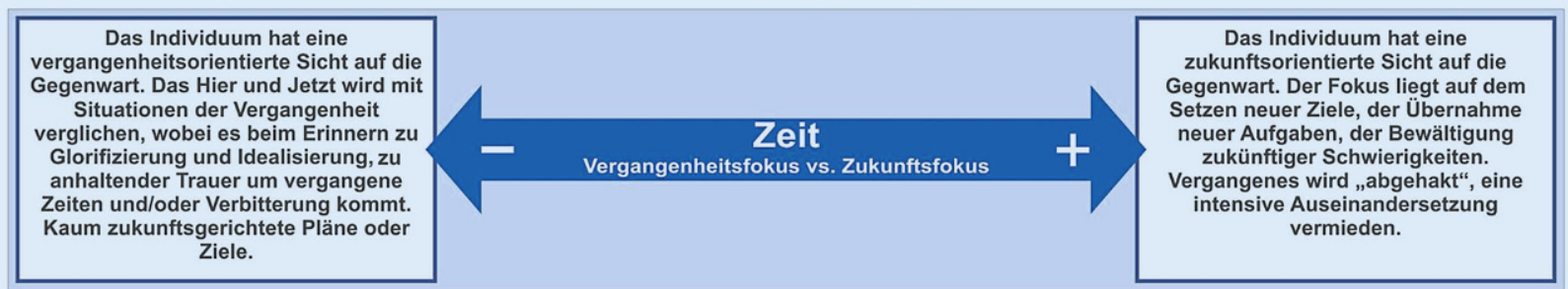

Zitat: „Ich habe ja in der NVA angefangen zu dienen und (...) da ist die Kameradschaft untereinander ganz, ganz doll gewesen, weil wir auch viel unterwegs gewesen sind. Von vier Wochen sind wir halt vierzehn Tage unterwegs gewesen, so. (...) wir haben uns, privat haben wir uns getroffen, die zwölf Leute, die wir in meiner Dienststellung gewesen sind ja? Und das ist halt, (...) ja das gibt es nicht mehr."
Zitat: „Für manch andere ist das mit dem Einsatz-Weiterverwendungs-Gesetz und den 30 Prozent und Berufssoldat vielleicht der richtige Weg, für mich ist es der falsche Weg. Ich will und werde rausgehen aus der Bundeswehr und in ein ziviles Leben zurückgehen, ja. Und ich freue mich auch drauf!" 
wieder, was als Hinweis sowohl auf die Qualität des Sampling der vorliegenden Studie als auch der Analyse zu sehen ist. Da einerseits Religion und Kirche in den USA eine andere Rolle spielen als in weiten Teilen Deutschlands, und die Abkehr von Religion und Glaube andererseits Folge schwerer Traumatisierung sein kann (Herman 2018), erscheint das „Fehlen“ dieser Kategorie innerhalb der hier untersuchten Gruppe plausibel.

Das entwickelte Modell deckt somit einen Großteil bestehender Coping-Kategorien und auch eine Vielzahl bekannter Abwehrmechanismen ab, verdeutlicht aber zusätzlich den Beziehungsaspekt und ermöglicht die Identifizierung des in diesem Zusammenhang neuen Bereichs der unbewussten, (inhaltlichen und/oder affektiven) zeitlichen Gebundenheit. Abschließend lässt sich sagen, dass nur durch die gewählte Art der Interviewführung, das Einbeziehen der Situation und des Gesprächszusammenhangs sowie den schul- und theorieoffenen Ansatz eine genaue Differenzierung zwischen emotionalem und reflexivem Umgang, das Erkennen der individuellen Motive, Abstufungen und Ausprägungen von beziehungs- und emotionenzulassendem bzw. -abwehrendem Verhalten sowie die Identifikation des zeitlichen Fokus möglich wurden.

Als ein die Therapieinanspruchnahme erschwerender Faktor ist die im Militär, insbesondere im Auslandseinsatz, möglicherweise besonders notwendige Fähigkeit, emotionale Verwundungen und die eigene Vulnerabilität zu verdrängen, teilweise auch zu leugnen, zu sehen. Die (totale) Kontrolle von Affekten könnte als notwendiger Teil der soldatischen Identität erlebt werden und einer das emotionale Erleben fördernden Psychotherapie im Weg stehen (Schuy et al. 2019). Inwiefern das Vermeiden emotionaler Situationen, die verminderte Wahrnehmung von Gefühlen und/oder eine verminderte Fähigkeit zur Emotionsregulation auch Teil einer Traumafolgesymptomatik waren, lässt sich innerhalb dieser Befragung nicht klären. Viele der befragten VeteranInnen berichteten eher von Mechanismen wie der Ausübung von Einzelsportarten, dem Konsum von Alkohol oder dem
Aggressionsabbau in Computerspielen statt von Handlungen, die über Bindung, Nähe und emotionale Verbundenheit zu sozialer Einbindung führen würden. Diese ist bekanntermaßen ein starker protektiver Faktor in der Entwicklung von Traumafolgestörungen. Eben diese, meist unbewussten, Emotionen (und somit auch Nähe) abwehrenden Mechanismen stellen eine große Barriere bei der Therapiesuche bzw. dem Einlassen auf eine psychotherapeutische Beziehung dar und können die soziale Isolation und Einsamkeit verstärken.

\section{Stärken und Limitationen der Studie}

Es handelt sich bei der Untersuchung um eine Pilotstudie zur Identifizierung sowie zur modellhaften Veranschaulichung von bewussten und unbewussten Bewältigungsmechanismen ehemaliger SoldatInnen mit Einsatzerfahrung. Mit 43 Interviews hat die Studie eine für ein qualitatives Design breite Datenbasis. Aufgrund der hohen medialen Resonanz (der Aufruf zur Teilnahme an der Studie wurde online innerhalb der ersten Woche allein 28.000 geteilt) sowie der zusätzlichen Rekrutierung im Bundeswehrkrankenhaus und innerhalb des Militärs dürfte eine sehr große Zahl ehemaliger SoldatInnen erreicht und durch das differenzierte Sampling eine große Variabilität kontrastierender Fälle eingeschlossen worden sein. Untersuchereinflüsse und daraus resultierende psychodynamische Prozesse konnten durch das Sampling, die divergente Teamzusammensetzung (ärztliche und psychologische MitarbeiterInnen beiderlei Geschlechts mit und ohne Militärund Einsatzerfahrung, mit und ohne psychotherapeutische Ausbildung unterschiedlicher Art) minimiert werden. Auch konnten sie durch den bewussten Umgang mit Gegenübertragungserleben sowie regelmäßige Teamintervision und -supervision analysiert und in die Auswertung einbezogen werden. Die offenen Interviews ermöglichten aufgrund ihrer geringen Vorgaben und der Möglichkeit späterer Nachfragen sehr detaillierte, individuelle, subjektbezogene Schilderungen der inneren und äußeren
Vorgänge. Durch die zusätzliche Analyse von unbewussten Mechanismen, die direkt im Gesprächsverlauf deutlich wurden, konnte einem bewussten oder unbewussten Selektions- bzw. Verzerrungsprozess, wie er in jeder Form von (retrospektiver) Befragung auftritt, entgegengewirkt werden.

Großer Wert wurde auf ein differenziertes Sampling mit dem Ziel der höchstmöglichen Heterogenität gelegt; bei der Datenerhebung und -analyse wurde sich stets an den Qualitätskriterien von Stamer et al. (2015) orientiert. Trotzdem muss die Generalisierbarkeit im Sinne einer Repräsentativität im statistischen Sinne, wie generell bei jeder Form qualitativen Vorgehens, nicht gegeben sein. Zudem handelt es sich um das erste Modell einer Pilotstudie. Seine Validierung bzw. kritische Überprüfung auch hinsichtlich seiner praktischen Nutzbarkeit muss und sollte in Folgestudien erfolgen.

\section{Fazit und Ausblick}

Das dargestellte Modell dient der Veranschaulichung eines gemeinsamen, Psychotherapieschulen und -strömungen verbindenden Denkansatzes, um im Rahmen diagnostischer Gespräche eine erste Einschätzung der bewussten und unbewussten Bewältigungsmöglichkeiten des Gegenübers treffen zu können. Dabei soll auf den Einsatz weiterer Fragebogen oder Selbst-Rating-Inventare verzichtet werden und die Einschätzung unabhängig von der psychotherapeutischen Prägung erfolgen können. Die große individuelle Variabilität der von den ehemaligen SoldatInnen zu verschiedenen Zeitpunkten eingesetzten Mechanismen bestätigt die These, dass die Situation und die empfundene Bedrohung Einfluss auf die „Wahl“ der CopingMechanismen haben. Dennoch komme es meistens zum simultanen Gebrauch verschiedener Mechanismen (Olff et al. 2005). Andererseits konnten bei den Teilnehmern individuell wiederkehrende, meist unbewusste Mechanismen identifiziert werden, die weitestgehend situationsunabhängig eingesetzt wurden und die für eine Konstanz persönlicher Kernmechanismen sprechen. Dies deckt sich mit der psychoanalytischen Annah- 
me eines mit der psychischen Struktur verknüpften „Abwehrprofils“ und auch mit Befunden der aktuellen Traumaforschung, nach denen der individuelle Bewältigungsstil Einfluss darauf habe, ob ein Mensch ein Trauma langfristig erfolgreich verarbeitet oder eine Traumafolgestörung entwickelt (Chang et al. 2003; Johnsen et al. 2002).

Weiterführende Forschung über bewusste und unbewusste Bewältigungsmechanismen (bei Traumafolgestörungen) ist unabhängig von den vorgestellten Ergebnissen nötig. Dies trifft in besonderem Maß auf einsatzgeschädigte (ehemalige) BundeswehrsoldatInnen zu, deren Zahl angesichts der globalen und politischen Entwicklungen in der $\mathrm{Zu}$ kunft vermutlich weiter ansteigen wird. Aus Sicht der Versorgungsforschung könnte beispielsweise die Identifizierung individueller Kernmechanismen im Sinne einer typologischen Einordnung zu verschiedenen Untersuchungszeitpunkten den Einfluss traumatischer Erlebnisse auf den persönlichen Bewältigungsstil beleuchten. Dies könnte langfristig zur Erstellung spezifischer Therapieangebote und einer differenzierteren Auseinandersetzung mit der Frage der persönlichen Vulnerabilität genutzt werden.

\section{Korrespondenzadresse}

\section{Dr. med. Loni Brants}

Klinik für Psychiatrie und Psychotherapie, Charite - Universitätsmedizin Berlin, Campus Charité Mitte

Chariteplatz 1, 10117 Berlin, Deutschland lonibrants@yahoo.de

Förderung. Das Projekt wurde durch Zuwendungen durch das Bundesministerium der Verteidigung finanziert.

Funding. Open Access funding provided by Projekt DEAL.

\section{Einhaltung ethischer Richtlinien}

Interessenkonflikt. L. Brants, K. Schuy, S. Dors, M. Horzetzky, H. Rau, P.L. Zimmermann, A. Ströhle und S. Siegel geben an, dass kein Interessenkonflikt besteht.

Alle beschriebenen Untersuchungen am Menschen oder an menschlichem Gewebe wurden mit Zustimmung der zuständigen Ethikkommission, im Einklang mit nationalem Recht sowie gemäß der Deklaration von Helsinki von 1975 (in der aktuellen, überarbeite- ten Fassung) durchgeführt. Das Einverständnis der Ethikkommission der Charité Berlin liegt vor. Von allen beteiligten Patienten liegt eine Einverständniserklärung vor.

Open Access. Dieser Artikel wird unter der Creative Commons Namensnennung 4.0 International Lizenz veröffentlicht, welche die Nutzung, Vervielfältigung, Bearbeitung, Verbreitung und Wiedergabe in jeglichem Medium und Format erlaubt, sofern Sie den/die ursprünglichen Autor(en) und die Quelle ordnungsgemäß nennen, einen Link zur Creative Commons Lizenz beifügen und angeben, ob Änderungen vorgenommen wurden.

Die in diesem Artikel enthaltenen Bilder und sonstiges Drittmaterial unterliegen ebenfalls der genannten Creative Commons Lizenz, sofern sich aus der Abbildungslegende nichts anderes ergibt. Sofern das betreffende Material nicht unter der genannten Creative Commons Lizenz steht und die betreffende Handlung nicht nach gesetzlichen Vorschriften erlaubt ist, ist für die oben aufgeführten Weiterverwendungen des Materials die Einwilligung des jeweiligen Rechteinhabers einzuholen.

Weitere Details zur Lizenz entnehmen Sie bitte der Lizenzinformation auf http://creativecommons.org/ licenses/by/4.0/deed.de.

\section{Literatur}

Beutel M (1990) Coping und Abwehr - Zur Vereinbarkeit zweier Konzepte. In: Muthny FA (Hrsg) Krankheitsverarbeitung. Hintergrundtheorien, klinische Erfassung und empirische Ergebnisse. Springer, Heidelberg

Brants L et al (2018) Abwehr und Coping ehemaliger Bundeswehrsoldaten in kritischen Lebensphasen. Symposiumsbeitrag der DeGPT-Konferenz 2018. Der ehemalige Soldat in der deutschen Gesellschaft' (DeGPT), Dresden

Carver CS, Scheier MF, Weintraub JK (1989) Assessing coping strategies: a theoretically based approach. JPers Soc Psychol 56(2):267-283

Chang C-M, LeeL-C,ConnorKM, Davidson JRT,JeffriesK, Lai T-J (2003) Posttraumatic distress and coping strategies among rescue workers after an earthquake. J Nerv Ment Dis 191:391-398

Chapman AL, Hadfield M, Chapman CJ (2015) Qualitative research in healthcare: an introduction to grounded theory using thematic analysis. JRColl Physicians 45:201-205

Cierpka M (Hrsg) (2014) Operationalisierte Psychodynamische Diagnostik OPD-2. Das Manual für Diagnostik und Therapieplanung, 3. Aufl. Huber, Bern (Arbeitskreis zur Operationalisierung PsychodynamischerDiagnostik)

Corbin JM, Strauss AL (2008) Basics of qualitative research. Techniques and procedures for developing grounded theory, 3. Aufl. SAGE, Los Angeles

Dresing T, Pehl T (2015) Praxisbuch: Interview, Transkription \& Analyse. Anleitungen und Regelsysteme für qualitativ Forschende. Anleitungen und Regelsysteme für qualitativ Forschende, 6 . Aufl., Eigenverlag, Marburg

Freud A (2006) Das Ich und die Abwehrmechanismen, 19. Aufl. S. Fischer, Frankfurt a. M. (Erste Auflage 1936)

Gigerenzer G, Kober H (2009) Bauchentscheidungen. Die Intelligenz des Unbewussten und die Macht der Intuition, 10. Aufl. Goldmann, München
Guest G, MacQueen KM, Namey EE (2012) Introduction to applied thematic analysis. In: Applied thematic analysis. SAGE, Thousand Oaks, S3-20

Hentschel U, Draguns JG, Ehlers W, Smith G (2004) Defense mechanisms: Current approaches to research and measurement. In: Hentschel $U$, Smith G, Draguns JG, Ehlers W (Hrsg) Advances in psychology, 136. Defense mechanisms. Theoretical, research and clinical perspectives. Elsevier, Oxford, S3-41

Herman J (2018) Die Narben der Gewalt. Traumatische Erfahrungen verstehen und überwinden $\mathrm{Bd}$. 5 . Junfermann, Paderborn, S63

Horr NK, Braun C, Volz KG (2014) Feeling before knowing why: the role of the orbitofrontal cortex in intuitive judgments - an MEG study. Cogn Affect Behav Neurosci 14(4):1271-1285. https:// doi.org/10.3758/s13415-014-0286-7

Johnsen BH, Eid J, Laberg JC, Thayer JF (2002) The effect of sensitization and coping style on post-traumatic stress symptoms and quality of life. Two longitudinal studies. Scand J Psychol 43(2):181-188. https://doi.org/10.1111/14679450.00285

Kato T (2015) Frequently used coping scales: a metaanalysis. Stress Health 31(4):315-323. https:// doi.org/10.1002/smi.2557

König K (1997) Abwehrmechanismen. Vandenhoeck \& Ruprecht, Göttingen

Kowalski JT, Hauffa R, Jacobs H, Höllmer H, Gerber WD, Zimmermann P (2012) Deployment-related stress disorder in german soldiers: utilization of psychiatric and psychotherapeutic treatment. Dtsch Arztebl Int 109(35-36):569-575. https:// doi.org/10.3238/arztebl.2012.0569

Laughlin HP (1979) The ego and its defenses, 2. Aufl. Aronson, New York

Lazarus RS (2000) Toward better research on stress and coping. Am Psychol 55(6):665-673. https://doi. org/10.1037//0003-066X.55.6.665

Lazarus RS, Folkman S (1984) Stress, appraisal and coping. Springer, New York

Lazarus RS, Launier R (1981) Stressbezogene Transaktionen zwischen Personen und Umwelt. In: Nitsch JR, Allmer H (Hrsg) Theorien, Untersuchungen, Maßnahmen. Huber, Bern, S213-259

Olff M, Langeland W, Gersons BPR (2005) The psychobiology of PTSD: coping with trauma. Psychoneuroendocrinology 30(10):974-982. https://doi.org/10.1016/j.psyneuen.2005.04. 009

Schuy K, Brants L, Dors S, Horzetzky M, Willmund G, Zimmermann P, Ströhle A, Rau H, Siegel S (2019) Psychological stigma costs as barriers to healthcare use in former soldiers of the German Armed Forces: a qualitative analysis. Mil Psychol 31(4):279-291.https://doi.org/10.1080/ 08995605.2019.1598228

Schwarzer R (1998) Stress and coping resources. Theory and review. In: Schwarzer R (Hrsg) Advances in Health Psychology Research. Freie Universität, Berlin (CDROM)

Seiffge-Krenke I (2017) Widerstand, Abwehr und Bewältigung (Psychodynamik kompakt). German Edition: 77. Vandenhoeck\& Ruprecht, Göttingen

Siegel S, Rau H, Dors S, Brants L, Börner M, Mahnke M et al (2017) Barrieren der Inanspruchnahme von Psychotherapie ehemaliger Soldatinnen und Soldaten der Bundeswehr (Veteranen). Eine Expertenbefragung. Z Evid Fortbild Qual Gesundhwes. https://doi.org/10.1016/j.zefq. 2017.06.006

Stamer M, Güthlin C, Holmberg C, Karbach U, Patzelt C, Meyer T, Arbeitsgruppe Qualitative Methoden 
des Deutschen Netzwerks Versorgungsforschung (DNVF) e.V. (2015) Qualitative Studien in der Versorgungsforschung - Diskussionspapier, Teil 3: Qualität qualitativer Studien. Gesundheitswesen 77:966-975

Steffens W, Kächele H (1988) Abwehr und Bewältigung - Mechanismen und Strategien. Wie ist eine Integration möglich? In: Steffens W, Kächele H (Hrsg) Bewältigung und Abwehr. Beiträge zur Psychologie und Psychotherapie schwerer körperlicher Krankheiten. Springer, Heidelberg, S $1-50$

Vaillant GE (1971) The theoretical hierarchy of adaptive ego mechanisms. Arch Gen Psychiatry 24:107-118

Vaillant GE (1992) The ego and mechanisms of defense. American Psychiatric Press, Washington DC

Wittchen H-U, Schönfeld S, Kirschbaum C, Thurau C, Trautmann S, Steudte $S$ et al (2012) Traumatic experiences and posttraumatic stress disorder in soldiers following deployment abroad: how big is the hidden problem? Dtsch Arztebl Int 109(35-36):559-569

\section{Wie viel Abstand, wann?}

Bei der Begegnung mit anderen Menschen halten wir instinktiv eine gewisse soziale Distanz ein, die von verschiedenen Faktoren abhängt. Dieser Wohlfühlabstand wird von Menschen mit psychopathischen Tendenzen häufig verletzt.

Wissenschaftler der Johannes GutenbergUniversität Mainz (JGU) haben die soziale Interaktion von Personen mit psychopathischen Tendenzen in einem 3-D-Labor untersucht und ermittelt, welchen Abstand sie zu ihrem virtuellen Gegenüber halten. Die Ergebnisse zeigen, dass Menschen mit psychopathischen Tendenzen den Wohlfühlabstand mangelhaft regulieren, da sie nicht auf die sozialen Signale des Gegenübers achten. Sie machen keinen Unterschied zwischen einer Person mit freundlichem Gesichtsausdruck und einer anderen mit wütendem Gesichtsausdruck und halten jeweils etwa den gleichen Abstand - im Gegensatz zu Menschen ohne psychopathische Tendenzen. „Wie unsere Studie weiter ergab, zeigen psychopathische Menschen keine adäquate Vermeidungsreaktion. Dies erklärt auch ihr Verhalten", teilt Robin Welsch vom Psychologischen Institut der JGU mit.

Unter Psychopathie versteht man ein dauerhaft abweichendes Verhalten in Verbindung mit zwischenmenschlichen und emotionalen Defiziten. Dazu gehört auch die Neigung, Schaden oder Leid zu verursachen, indem gegen soziale Normen verstoßen wird. Klinische Berichte weisen in diesem Zusammenhang häufig darauf hin, dass psychopathische Personen den persönlichen Wohlfühlabstand missachten, indem sie zum Beispiel anderen auf unangenehme Weise zu nahe kommen. Wie sich die Abstandsregulation bei psychopathischen Menschen genau verhält, hat Welsch im Labor für virtuelle Realität der Abteilung Allgemeine Experimentelle Psychologie untersucht.

\section{Begegnung mit Avatar im virtuellen} Versuchslabor

Die studentische Stichprobe umfasste 76 Probanden, 51 Frauen und 25 Männer im Alter zwischen 19 und 38 Jahren. Psychopathische Tendenzen wurden anhand eines Fragebogens erfasst, der insbesondere die beiden Faktoren selbstzentrierte Impulsivität und furchtlose Dominanz ermittelt. Die Probanden wurden schließlich mit dem Setup im 3-D-Labor vertraut gemacht und dann den jeweiligen Tests unterzogen. „Das Labor bietet eine virtuelle Realität vergleichbar mit den 3-D-Bildern im Kino. Hinzukommt allerdings, dass das 3-D-Bild auf die Bewegung eines Versuchsteilnehmers reagiert und sich anpasst und damit den Probanden in die virtuelle Realität eintauchen lässt", erklärt Welsch. Im zweiten Versuchsteil konnten die Teilnehmerinnen und Teilnehmer außerdem den Avatar mit einem Joystick steuern.

\section{Angemessene Vermeidungsreaktion}

\section{bleibt aus}

Zeigt der Avatar einen freundlichen Gesichtsausdruck, gehen Menschen ohne psychopathische Tendenzen auf den Avatar zu, bis etwa 1,00 bis 1,10 Meter Abstand besteht. Im Falle eines wütenden Gesichtsausdrucks halten sie eine Distanz von 1,25 Meter ein. Diesen Unterschied machen Menschen mit psychopathischen Neigungen nicht, sie treten unabhängig vom Gesichtsausdruck auf 1,10 Meter heran. Im zweiten Experiment sollten die Probanden in Reaktion auf die Mimik des Avatars einen Joystick bewegen. „Wir stellten fest, dass die Versuchsteilnehmer mit psychopathischen Tendenzen keine angemessene Vermeidungsreaktion zeigen, obwohl sie den Gesichtsausdruck des Avatars richtig deuten können", so Welsch, wissenschaftlicher Mitarbeiter und Erstautor der Studie, an der außerdem Dr. Christoph von Castell und Prof. Dr. Heiko Hecht beteiligt sind. Zusammenfassend kann man also sagen, dass Psychopathie nicht einfach zu einer unangemessenen sozialen Distanz führt, sondern dass lediglich die situationsangemessene Regulation der sozialen Distanz gestört ist.

Originalpublikation: Robin Welsch, Christoph von Castell, Heiko Hecht: Interpersonal Distance Regulation and Approach-Avoidance Reactions Are Altered in Psychopathy Clinical Psychological Science, 26. November 2019; DOI: 10.1177/2167702619869336

Quelle: Johannes GutenbergUniversität Mainz (https://www.unimainz.de/presse/aktuell/; 16.04.2020) 